\title{
Evolution of the characteristics of Parametric X-ray Radiation from textured polycrystals under different observation angles
}

\author{
V.I. Alekseev $^{\mathrm{a}, \mathrm{b}}$, A.N. Eliseyev ${ }^{\mathrm{a}, \mathrm{b}}$, E. Irribarra ${ }^{\mathrm{c}}$, I.A. Kishin ${ }^{\mathrm{a}, \mathrm{b}}$, A.S. Klyuev ${ }^{\mathrm{b}}$, \\ A.S. Kubankin ${ }^{a, b, *}$, R.M. Nazhmudinov ${ }^{a, b}$, P.N. Zhukova ${ }^{b}$ \\ a P.N. Lebedev Physical Institute RAS, 308007, Moscow, Russia \\ b Belgorod National Research University, 119991, Belgorod, Russia \\ c Escuela Politécnica Nacional, E11-253, Quito, Ecuador
}

\section{A R T I C L E I N F O}

\section{Article history:}

Received 6 September 2017

Received in revised form 17 November 2017

Accepted 17 December 2017

Available online 20 December 2017

Communicated by V.A. Markel

\section{Keywords:}

Parametric X-ray Radiation

Textured polycrystal

Rocking curve

Polarization bremsstrahlung

Spectral-angular characteristics

\begin{abstract}
A B S T R A C T
The Parametric X-Ray radiation (PXR) spectra and yield dependencies on the orientation angle are measured during the interaction of $7 \mathrm{MeV}$ electrons with a tungsten textured polycrystalline foil for different observation angles. The effects of PXR spectral density increase and PXR yield orientation dependence broadening in the backward direction is shown experimentally for the first time. The experimental results are compared with PXR kinematical theories for both mosaic crystals and polycrystals.
\end{abstract}

(c) 2017 Elsevier B.V. All rights reserved.

\section{Introduction}

PXR is generated due to the coherent scattering of the Coulomb field of fast charged particles on atomic structures. The spectral and angular characteristics of PXR are determined by the properties of the incident charged particle, the parameters of the target and the interaction process geometry. PXR from crystals [1-4] and polycrystals with random oriented grains [5,6] has been deeply studied theoretically and experimentally observed for different conditions.

On the other hand, PXR from textured polycrystals (polycrystals which have a predominant orientation of grains), has been described in [7] only by a model based on the PXR kinematical theory for mosaic crystals. In that work, the texture pattern of a polycrystal was considered as the crystal mosaic parameter. However, a clear theory does not exist.

PXR from textured polycrystals was observed in [8,9] during the interaction of $150 \mathrm{MeV}$ electrons with a molybdenum foil at observation angles of $11.27^{\circ}$ and $25.89^{\circ}$. Intensive PXR peaks comparable to the $K_{\beta}$ Characteristic X-ray Radiation (CXR) line were registered. Therefore, PXR from textured polycrystalline foils is re-

\footnotetext{
* Corresponding author.

E-mail address: kubankin@bsu.edu.ru (A.S. Kubankin).
}

garded as an alternative to produce intensive quasimonochromatic X-Ray beams.

PXR from textured polycrystals was also observed in $[7,10]$ during the interaction of $7 \mathrm{MeV}$ electrons with a tungsten foil for the observation angle of $180^{\circ}$ (backward geometry). In contrast with [8], it was reported that the PXR peak energy depends on the target orientation angle. The experimental results and the developed model have showed a good agreement [7].

The experimental study of PXR is motivated not only for fundamental research but also for possible applications [3,11,12]. For instance, the spectral and angular characteristics of PXR can be used for medium diagnostics because of their strong dependence on the crystalline structure of the sample. Theoretical works predict the possibilities to use PXR to measure the parameters of the elementary lattice of crystalline structures [13], grain size of polycrystalline materials [13-15], distribution of the crystalline blocks on the orientation angles for textured polycrystals [16] and crystals mosaicity [17].

An important result for medium structure diagnostics was presented in [18], where it was shown that the spectral width of PXR peaks from polycrystals with random oriented grains is proportional to $\gamma^{-2}$ ( $\gamma$-Lorenz factor of the exciting radiation particle) and the peaks amplitude is proportional to $\gamma^{2}$ for the backward geometry while the same characteristics are proportional to $\gamma^{-1}$ and $\gamma$ when the observation angle is far from $\pi$. This feature al- 


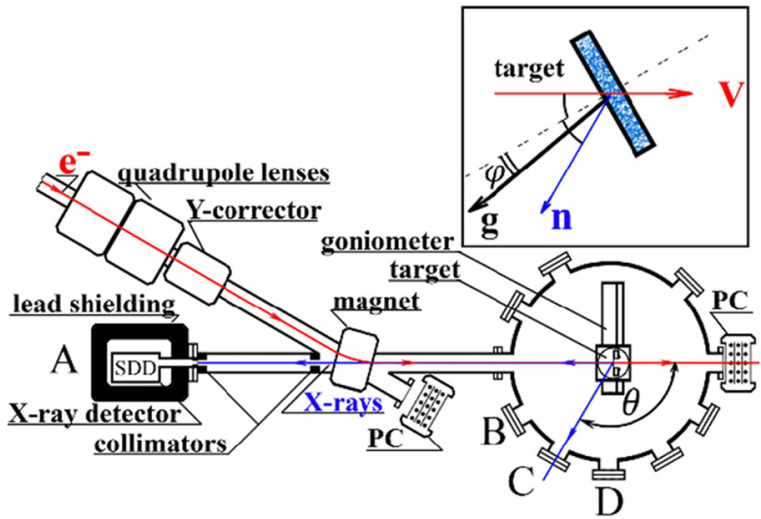

Fig. 1. Experimental setup, the detector was installed in positions $A, B, C, D$; PC - Proportional Chamber; $\mathbf{g}$ - predominant reciprocal lattice vector of the (200) plane; $\mathbf{V}$ - electron velocity; $\mathbf{n}$ - direction of radiation registration.

lows one to increase the resolution of the possible PXR application for medium structure diagnostics because the PXR peaks become narrow and high.

Nevertheless, a detailed comparison of experimental data with theory should be perform before PXR can be applied as a tool for medium structure diagnostics. It is important to emphasize that the textured polycrystalline structures are the most common state of the metals.

This work presents the experimental measurements of PXR produced during the interaction of relativistic electrons with a textured polycrystalline foil for different observation angles including the backward geometry. The evolution of the spectral peak parameters is fixed. The results allow one to perform the first detailed verification of the PXR kinematic theories for crystals and polycrystals. The similarities and differences between the mentioned theories are highlighted.

\section{Experiment}

The experiment was performed in the department of High Energy Physics of the Lebedev Physical Institute. The experimental setup presented in Fig. 1 was described in detail in [19]. A $7 \mathrm{MeV}$ beam of electrons with a divergence smaller than 2 mrad and a size smaller than $3 \mathrm{~mm}$ at the target position, interacted with the $20 \mu \mathrm{m}$ thick tungsten textured polycrystalline foil used in [7]. It was established that the (200) plane has a predominant orientation parallel to the target surface as a result of the rolling process used during the target preparation [20]. The texture angular size (FWHM) equals $5.4^{\circ}$. The target was installed in a goniometer with 2 degrees of freedom which controls the orientation angle $\varphi$ with an accuracy of $0.01^{\circ}$.

Two sets of experiments were performed, the first was intended to determine the dependencies of the spectral width and intensity of the (200) PXR peak on the observation angle $\theta$; four values of $\theta$ were considered: $90.8^{\circ} \pm 1.5^{\circ}, 119.6^{\circ} \pm 0.5^{\circ}, 151.0^{\circ} \pm 1.6^{\circ}$ and $180.0^{\circ} \pm 0.5^{\circ}$. The measurements were performed near the specular condition $\left(\varphi=0^{\circ}\right)$ of the averaged position of the (200) crystallographic plane regarding to the incident electrons and the detector position. The second set of experiments consisted of the measurements of the dependencies of the PXR yield for the (200) plane on the orientation angle $\varphi$ (rocking curve). The measurements were performed under observation angles of $90.8^{\circ}$ and $180.0^{\circ}$.

The radiation was registered by a silicon drift detector which energy resolution for the experimental conditions was $149 \mathrm{eV}$ at the $5.9 \mathrm{keV}$ energy. The detector collimation angle was $1.8 \cdot 10^{-7} \mathrm{sr}$ for the backward geometry and $2.1 \cdot 10^{-5} \mathrm{sr}$ for the other observation angles. It should be noted that the typical angular size value

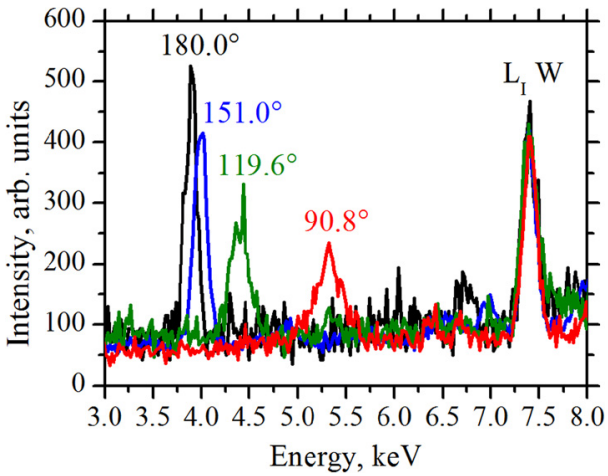

Fig. 2. PXR peaks from the tungsten textured polycrystal for different observation angles.

$7.6^{\circ}$ of the PXR cone (under condition $\gamma^{-2} \gg \omega_{0}^{2} / \omega^{2}$ when the density effect is suppressed; $\omega_{0}$-plasma frequency; $\omega$ - the energy of PXR photons) for $7 \mathrm{MeV}$ electrons is comparable with the target texture size.

\section{Results and discussion}

The measured PXR spectra for the (200) plane normalized on the number of counts of the tungsten CXR $L_{l}$ line for different observation angles are presented in Fig. 2. It can be observed the evolution of the PXR peak characteristics depending on the observation angle. It is clearly observed that the position, amplitude and spectral width of the PXR peak depend on the observation angle. The peak amplitude increases, and the spectral width decreases when $\theta$ approaches to $\pi$. It is the manifestation of both effects simultaneously what allows one to affirm that an increase of the PXR spectral density was observed for the first time. This behavior coincides qualitatively with the theoretical predictions of [18]. In previous works, this theory was partially validated for an isolated observation angle $-\pi[19,21]$ but a dynamical approval was not performed and the evolution of the PXR characteristics was not observed.

A possible contribution of diffracted free X-rays related to bremsstrahlung or transition radiation can be neglected based on the results of work [7]. Consequently, it is important to emphasize that the nature of the observed peaks is related only to the PXR mechanism.

To analyze the PXR characteristics quantitatively the experimental results are compared with [18] considering the detector energy resolution under experimental conditions, which depends on the detector setups and the registered photons energy.

The experimental energy resolution was determined based on measurements of the FWHM of Characteristic X-ray (CXR) peaks since the real spectral width of those peaks constitutes some eV in the analyzed energy region [22]; additionally, it is smaller than the nominal detector energy resolution. Fig. 3 presents the comparison of the measured spectra with theories [18] (dash line) and [7] (solid line).

The disagreement of experimental data with [18] is evident for the four observation angles. It concerns position, FWHM and shape of the peak.

The divergence between the experimental and theoretical peak positions has an order of magnitude of $\mathrm{eV}$. It is related to the influence of the target orientation $\left(\varphi=3^{\circ}\right)$ on the energy of the PXR peak position reported in [7]. Additionally, it is important to highlight that theory [18] was developed for a target which consists of randomly oriented grains and the radiation characteristics do not depend on the orientation angle between the electron beam and the target surface. This condition is not fulfilled for the analyzed textured polycrystalline foil, since a predominant orientation 


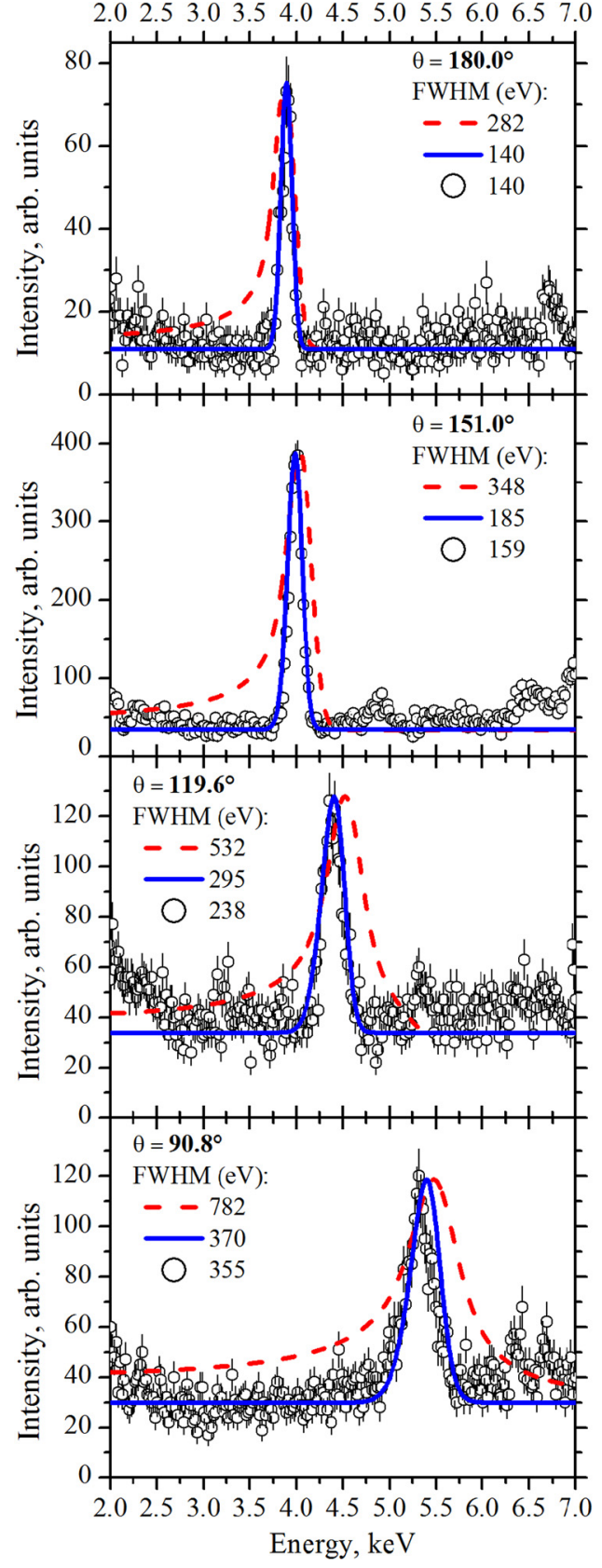

Fig. 3. Comparison of experimental results with the theory of PXR for polycrystals (dash line) [18] and the model of PXR for mosaic crystals (solid line) [7].

of the grains does exist (texture pattern). Consequently, the PXR energy depends on the orientation angle [7].

Another reason that could influence on the divergence is that the theory [18] is most suitable for targets consisting of light elements $z<20$ while in the performed experiment $z=74$. Finally, it can be observed that the FWHM of the theoretical curve (dash line) is considerably wider than the experimental value. This occurs because the PXR peak from randomly oriented polycrystals [18] is wider than the one from perfect crystals [23] while textured polycrystals are in between those structures. The mentioned reasons contribute to the divergence between the theoretical and experimental curves.

Considering the crystalline structure of the target, the model proposed in [7] describes better the observed results. In the model, the mosaic parameter of the crystal $\sigma_{\|}=\sigma_{\perp}$ is regarded as the texture size of the polycrystalline sample $5.4^{\circ}$. The orientation angle

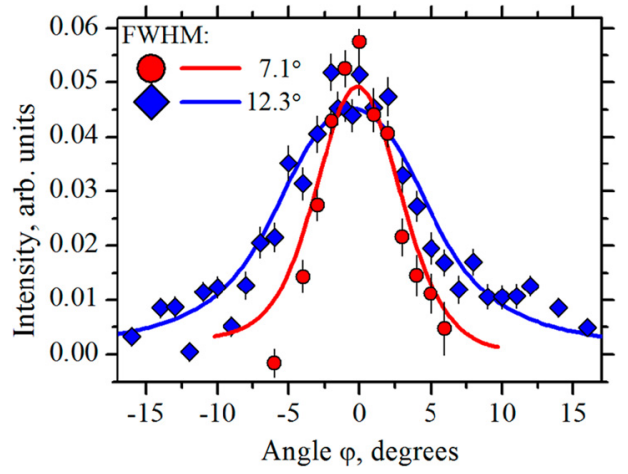

Fig. 4. Dependence of the PXR yield for the (200) plane on the orientation angle $\varphi$ measured for observation angles of $90.8^{\circ}$ (circles) and $180.0^{\circ}$ (rhombus). The lines represent the theoretical curves calculated according to [7].

is set to $3^{\circ}$. Finally, the electron multiple scattering angle was calculated for an electron trajectory equal to the attenuation length for the (200) peak energy according to the observation angle. It can be observed that the model presented in Fig. 3 (solid line) fits the experimental points within the frame of the statistical error.

The results of the second set of experiments, consisting of the measurements of the PXR yield dependence on the orientation angle, for observation angles $\theta=90.8^{\circ}$ and $\theta=180^{\circ}$ are presented in Fig. 4.

It can be observed that the dependence of the PXR yield on the orientation angle from textured polycrystals, is not similar neither to that one of a crystal [2] nor to that one of a polycrystal [5]. The orientation dependence of PXR from perfect crystals presents two peaks at values $\pm \sqrt{\gamma^{-2}+\omega_{0}^{2} / \omega^{2}} / 2$ relative to the specular condition of the electron beam axis, crystallographic plane and direction of PXR observation [4]. On the other hand, the PXR orientation dependence from polycrystals with random orientation of grains is a constant which depends only on the absorption properties of the sample [5]. Consequently, a textured polycrystal represents a transition state between those structures.

The experimental points were fitted by the Gaussian function (not shown in Fig. 4) to determine the FWHM of the distributions. The results clearly show that the orientation dependence for $\theta=$ $90.8^{\circ}\left(\mathrm{FWHM}=5.7^{\circ}\right)$ is approximately 1.7 times narrower than for $\theta=180^{\circ}\left(\mathrm{FWHM}=9.6^{\circ}\right)$. The observed difference between the FWHM of the orientation dependencies cannot be explained by the change of the PXR cone angular size $2 \sqrt{\gamma^{-2}+\omega_{0}^{2} / \omega^{2}}$, which depends on the PXR photons energy and consequently on the observation angle. The calculated difference between the PXR cone angular sizes, for the observed peak energies $\omega=5.321 \mathrm{keV}$ $\left(\theta=90.8^{\circ}\right)$ and $\omega=3.891 \mathrm{keV}\left(\theta=180^{\circ}\right)$, equals $0.16^{\circ}$ and it is substantially smaller than the observed difference $5.2^{\circ}$. Consequently, the observed effect can be explained only by the presence of texture and cannot be observed for perfect crystals and polycrystals with random orientation of the grains. This explanation is confirmed by the agreement between theory and experiment presented in Fig. 4.

\section{Conclusions}

Two new effects in the PXR mechanism were observed. The first is related to the observation of the predicted effect of PXR spectral peak anomalous transformation when the observation angle approaches to $\pi$. This effect qualitatively coincides with the theory presented in [18]: the PXR spectral density increases because the amplitude of PXR spectral peaks increases and their width decreases. The second result consists in the observation of texture influence on PXR orientation dependence width. 
The performed calculations show that PXR from textured polycrystals can be described by the kinematical theory of mosaic crystals, regarding the texture pattern of the polycrystalline sample as the mosaic parameter. On the other hand, a significant disagreement is observed between the PXR theory for polycrystals with random oriented grains.

The performed comparisons are essential in order to apply PXR in medium structure diagnostics [13-17].

\section{Acknowledgements}

The work was supported by a Program of the Ministry of Education and Science of the Russian Federation for higher education establishments, Project No. 3.1631.2017/ПЧ, by grant 16-32-00502 mol-a from the Russian Foundation for Basic Research and by the project No. PIJ-16-03 of the Escuela Politécnica Nacional.

\section{References}

[1] M.L. Ter-Mikaelian, High Energy Electromagnetic Processes in Condensed Media, 1972.

[2] V.G. Baryshevsky, I.D. Feranchuk, Parametric X-rays from ultrarelativistic electrons in a crystal: theory and possibilities of practical utilization, J. Phys. France 44 (1983) 913-922.

[3] V.G. Baryshevsky, I. Feranchuk, A. Ulyanenkov, Parametric X-Ray Radiation in Crystals, Springer-Verlag, Berlin, Heidelberg, 2005

[4] A.P. Potylitsyn, Electromagnetic Radiation of Electrons in Periodic Structures, Springer, Berlin, Heidelberg, 2011.

[5] N.N. Nasonov, Collective effects in the polarization bremsstrahlung of relativistic electrons in condensed media, Nucl. Instrum. Methods Phys. Res., Sect. B, Beam Interact. Mater. Atoms 145 (1998) 19-24.

[6] S. Blazhevich, A. Chepurnov, V. Grishin, B. Ishkhanov, N. Nasonov, V. Petukhov, et al., Polarization bremsstrahlung of relativistic electrons in aluminium, Phys. Lett., Sect. A, Gen., At. Solid State Phys. 254 (1999) 230-232.

[7] V.I. Alexeyev, A.N. Eliseyev, E. Irribarra, I.A. Kishin, A.S. Kubankin, R.M. Nazhmudinov, Observation of parametric X-ray radiation in an anomalous diffraction region, Phys. Lett. A 380 (2016) 2892-2896.

[8] Y. Takabayashi, I. Endo, K. Ueda, C. Moriyoshi, A.V. Shchagin, Observation of intense PXR from textured polycrystal, Nucl. Instrum. Methods Phys. Res., Sect. B, Beam Interact. Mater. Atoms 243 (2006) 453-456.
[9] S. Nawang, I. Endo, M. Iinuma, T. Takahashi, A. Kohara, K. Ueda, et al., Parametric X-ray study from textured molybdenum polycrystal, J. Phys. Soc. Jpn. (2006) 124705

[10] V.I. Alekseev, A.N. Eliseev, E.F. Irribarra, I.A. Kishin, A.S. Kubankin, R.M. Nazhmudinov, et al., Research of the polarization bremsstrahlung of relativistic electrons in polycrystalline targets, Nucl. Instrum. Methods Phys. Res., Sect. B, Beam Interact. Mater. Atoms 342 (2015) 47-51.

[11] G. Kube, C. Behrens, A.S. Gogolev, Y.P. Popov, A.P. Potylitsyn, W. Lauth, et al. Investigation of the applicability of parametric X-ray radiation for transverse beam profile diagnostics, in: IPAC 2013: Proceedings of the 4th International Particle Accelerator Conference, 2013, pp. 491-493.

[12] Y. Takabayashi, Parametric X-ray radiation as a beam size monitor, Phys. Lett. A 376 (2012) 2408-2412.

[13] N. Nasonov, P. Zhukova, V. Sergienko, Polarization bremsstrahlung in a backward direction for medium structure diagnostics, J. Phys. Conf. Ser. 236 (2010) 012017.

[14] N.N. Nasonov, V.A. Nasonova, I.G. Popov, Polarization bremsstrahlung from relativistic electrons moving in a small-grained medium, Phys. At. Nucl. 64 (2001) 966-970.

[15] A.V. Shchagin, Possibilities for measurement of nano-crystallites size with use of parametric X-ray radiation, J. Phys. Conf. Ser. 236 (2010) 012020.

[16] I. Lobach, A. Benediktovitch, I. Feranchuk, A. Lobko, Parametric X-rays from polycrystalline target, Nucl. Instrum. Methods Phys. Res., Sect. B, Beam Interact. Mater. Atoms 360 (2015) 75-80.

[17] A.S. Kubankin, N.N. Nasonov, On the possibility of using parametric X-ray radiation to study anisotropy of a crystal mosaic structure, J. Surf. Investig.: X-Ray Synchrotron Neutron Tech. 2 (2008) 317.

[18] V. Astapenko, N. Nasonov, P. Zhukova, Anomalous peak in the spectrum of polarizational bremsstrahlung from relativistic electrons moving through a solid target, J. Phys. B, At. Mol. Opt. Phys. 40 (2007) 1337-1346.

[19] V.I. Alexeyev, V.A. Astapenko, A.N. Eliseyev, E.F. Irribarra, V.A. Karpov, I.A Kishin, et al., Investigation into the mechanisms of X-ray generation during the interaction between relativistic electrons and a medium by means of the Röntgen-1 setup, J. Surf. Investig.: X-Ray Synchrotron Neutron Tech. 11 (2017) 694-698.

[20] H. Wenk, P.V. Houtte, Texture and anisotropy, Rep. Prog. Phys. 67 (2004) 1367.

[21] V.I. Alekseev, K.A. Vokhmyanina, A.N. Eliseev, P.N. Zhukova, A.S. Kubankin, R.M. Nazhmudinov, et al., Measuring coherent peaks of polarization bremsstrahlung from relativistic electrons in polycrystalline targets in backscattering geometry, Tech. Phys. Lett. 38 (2012) 294-296.

[22] M.O. Krause, J.H. Oliver, Natural widths of atomic K and L levels, K $\alpha$ X-ray lines and several KLL Auger lines, J. Phys. Chem. Ref. Data 8 (1979) 329-338.

[23] K. Brenzinger, B. Limburg, H. Backe, S. Dambach, H. Euteneuer, F. Hagenbuck, et al., How narrow is the linewidth of parametric X-ray radiation?, Phys. Rev. Lett. 79 (1997) 2462-2465. 